\title{
ON THE ASYMPTOTIC BEHAVIOUR OF ASSOCIATED PRIMES OF GENERALIZED LOCAL COHOMOLOGY MODULES
}

\author{
KAZEM KHASHYARMANESH ${ }^{\varpi}$ and AHMAD ABBASI
}

(Received 15 November 2004; revised 24 July 2006)

Communicated by J. Du

\begin{abstract}
Let $M$ and $N$ be finitely generated and graded modules over a standard positive graded commutative Noetherian ring $R$, with irrelevant ideal $R_{+}$. Let $H_{R_{+}}^{k}(M, N)_{n}$ be the $n$th component of the graded generalized local cohomology module $H_{R_{+}}^{k}(M, N)$. In this paper we study the asymptotic behavior of $\operatorname{Ass}_{R_{+}}\left(H_{R_{+}}^{k}(M, N)_{n}\right)$ as $n \longrightarrow-\infty$ whenever $k$ is the least integer $j$ for which the ordinary local cohomology module $H_{R_{+}}^{j}(N)$ is not finitely generated.
\end{abstract}

2000 Mathematics subject classification: primary 13D45, 13A02, 13E05; secondary 14B15.

\section{Introduction}

There is a lot of current interest in the theory of graded local cohomology modules and in recent years there have appeared many papers concerned with this context and its developments. The main purpose of this paper is to establish an asymptotic behaviour of associated prime ideals of graded components of generalized local cohomology modules.

The concept of generalized local cohomology of $R$-modules $M$ and $N$ relative to an ideal $I$ of a commutative Noetherian ring $R$ was introduced by Herzog in [10] and studied by Suzuki in [21] (see also [2]) as

$$
H_{I}^{i}(M, N)=\underset{n \in \mathbb{N}_{0}}{\lim } \operatorname{Ext}_{R}^{i}\left(M / I^{n} M, N\right),
$$

where $i \in \mathbb{N}_{0}$, and was studied in $[1,11,14,16,22,23]$ (here $\mathbb{N}_{0}$ and $\mathbb{N}$ denote the set of non-negative and positive integers, respectively; $\mathbb{Z}$ will denote the set of all

(C) 2007 Australian Mathematical Society 1446-7887/07 \$A2.00+0.00 
integers). It is well known that the functor $H_{I}^{i}(-,-): C(R) \times C(R) \rightarrow C(R)$ is a two variable additive $R$-linear functor and for $M=R$, it is converted to $H_{l}^{i}(-)$, the $i$ th ordinary local cohomology functor (where $C(R)$ denotes the category of all $R$-modules and $R$-homomorphisms).

Assume that $R=\bigoplus_{n \in \mathrm{N}_{0}} R_{n}$ is a positively graded Noetherian ring which is standard, in the sense $R=R_{0}\left[R_{1}\right]$, and set $R_{+}=\bigoplus_{n \in \mathbb{N}} R_{n}$, the irrelevant ideal of $R$. Let $M=\bigoplus_{n \in \mathbb{Z}} M_{n}$ and $N=\bigoplus_{n \in \mathbb{Z}} N_{n}$ be non-zero finitely generated graded $R$ modules. It is well known that $H_{i}^{i}(N)$ is equipped with a natural grading for all $i \in \mathbb{N}_{0}$ (compare [8, Remarks 12.3.6]). The associated primes of graded components of local cohomology modules were studied in a number of papers (compare [3-7, 12,13,17, 18]). Brodmann and Hellus, in [6], showed that if $k$ is an integer such that $H_{R_{+}}^{i}(N)$ is finitely generated for all $i<k$, then the associated prime ideals of $H_{R_{+}}^{k}(N)_{n}$ is asymptotically stable as $n \longrightarrow-\infty$. Recall that we say $\operatorname{Ass}_{R_{0}}\left(H_{R_{+}}^{i}(N)_{n}\right)$ are asymptotically stable as $n \rightarrow-\infty$ if there exists an integer $n_{0} \in \mathbb{Z}$ such that $\operatorname{Ass}_{R_{0}}\left(H_{R_{+}}^{i}(N)_{n}\right)=\operatorname{Ass}_{R_{0}}\left(H_{R_{+}}^{i}(N)_{n_{0}}\right)$ for all $n \leq n_{0}$. Also, in [14], the first present author established the above result in the context of generalized local cohomology modules (see [14, Theorem 3.5]). We say that $\operatorname{Ass}_{R_{0}}\left(H_{R_{+}}^{i}(M, N)_{n}\right)$ is asymptotically stable as $n \longrightarrow-\infty$ if there exists an $n_{0} \in \mathbb{Z}$ such that $\operatorname{Ass}_{R_{0}}\left(H_{R_{+}}^{i}(M, N)_{n}\right)=\operatorname{Ass}_{R_{0}}\left(H_{R_{+}}^{i}(M, N)_{n_{0}}\right)$ for all $n \leq n_{0}$ (see [14, Notation and Remarks 3.3]). In this paper we will show that if $H_{R_{+}}^{i}(N)$ is finitely generated for all $i<k$ then $\operatorname{Ass}_{R_{0}}\left(H_{R_{+}}^{k}(M, N)_{n}\right)$ is asymptotically stable as $n \longrightarrow-\infty$.

The grading of the generalized local cohomology module $H_{R_{+}}^{i}(M, N)$ has been studied by the first present author in [14]. He found that the grading of the generalized local cohomology modules with respect to the irrelevant ideal of $R$ has some properties similar to the ordinary local cohomology modules. Let us now briefly recall some basic properties of graded generalized local cohomology modules (see [14]).

(i) For every finitely generated graded $R$-module $M$, if we forget the grading on the module ${ }^{*} \operatorname{Hom}_{R}(M, N)$ then we have the module $\operatorname{Hom}_{R}(M, N)$ (see [9, Pages 3233]). Hence there is a homogeneous isomorphism $H_{I}^{0}(M, N) \cong H_{I}^{0}\left(\operatorname{Hom}_{R}(M, N)\right)$ for every homogeneous ideal $I$ of $R$.

(ii) If there is a short exact sequence of graded modules and homogeneous homomorphisms

$$
0 \longrightarrow N^{\prime} \rightarrow N \longrightarrow N^{\prime \prime} \longrightarrow 0
$$

(by 'homogeneous" here we mean 'homogeneous of degree zero') then there exists the following long exact sequence of graded modules and homogeneous homomorphisms

$$
0 \longrightarrow H_{I}^{0}\left(M, N^{\prime}\right) \longrightarrow H_{I}^{0}(M, N) \longrightarrow H_{I}^{0}\left(M, N^{\prime \prime}\right) \longrightarrow H_{I}^{1}\left(M, N^{\prime}\right) \longrightarrow \cdots .
$$

(iii) Let $R^{\prime}=\oplus_{n \in N_{0}} R_{n}^{\prime}$ be a second commutative Noetherian ring and let 
$f: R \longrightarrow R^{\prime}$ be a homogeneous flat ring homomorphism. Let $I$ be a homogeneous ideal of $R$. Then there exists a homogeneous isomorphism

$$
H_{I}^{i}(M, N) \otimes_{R} R^{\prime} \cong H_{I R^{\prime}}^{i}\left(M \otimes_{R} R^{\prime}, N \otimes_{R} R^{\prime}\right)
$$

for all $i \in \mathbb{N}_{0}$.

(iv) If $N$ is a $R_{+}$-torsion $R$-module, then by choosing a *injective $R_{+}$-torsion resolution $E^{\bullet}$ on $N$ in which each term is an $R_{+}$-torsion $R$-module for all $i \in \mathbb{N}_{0}$, we obtain the following homogeneous isomorphisms (see [16, Lemma 2.2])

$$
\begin{aligned}
H_{R_{+}}^{i}(M, N) & =H^{i}\left(H_{R_{+}}^{0}\left({ }^{*} \operatorname{Hom}_{R}\left(M, E^{\bullet}\right)\right)\right)=H^{i}\left(\operatorname{Hom}_{R}\left(M, H_{R_{+}}^{0}\left(E^{\bullet}\right)\right)\right) \\
& \cong H^{i}\left(\operatorname{Hom}_{R}\left(M, E^{\bullet}\right)\right) \cong \operatorname{Ext}_{R}^{i}(M, N) .
\end{aligned}
$$

Moreover, by [14, Lemma 3.1], for all $i \in \mathbb{N}_{0}, H_{R_{+}}^{i}(M, N)_{n}$ is a finitely generated $R_{0}$-module and only finitely many of $H_{R_{+}}^{i}(M, N)_{n}$ can be non-zero.

Throughout the paper, let $R=\bigoplus_{n \in \mathbb{N}_{0}} R_{n}$ be a $\mathbb{N}_{0}$-graded standard commutative Noetherian ring. We will use the notation defined in this section throughout the paper.

\section{Asymptotic behavior of associated primes}

Let $N$ be a graded $R$-module and $I$ be a homogeneous ideal of $R$. A sequence of homogeneous elements $a_{1}, \ldots, a_{k}$ of $I$ is said to be a homogeneous $I$-filter regular sequence on $N$ if $a_{i} \notin \mathfrak{p}$ for all $\mathfrak{p} \in \operatorname{Ass}_{R}\left(N /\left(a_{1}, \ldots, a_{i-1}\right) N\right) \backslash V(I)$ for all $i=1, \ldots, k$, where $V(I)$ denotes the set of prime ideals of $R$ containing $I$. Also, a graded $R$-module $T=\bigoplus_{n \in \mathbb{Z}} T_{n}$ is said to be asymptotically gap free, if the cardinal number of

$$
\left\{n \in \mathbb{Z}_{\leq 0} \mid T_{n} \neq 0, T_{n+1}=0\right\}
$$

is finite (compare [6, Definition and Remark 4.1]).

PROPOSITION 2.1. Assume that $I$ is an ideal of $R$ generated by elements of positive degrees. Then, for every positive integer $n$, there exists a homogeneous I-filter regular sequence on $N$ of length $n$.

PROOF. Let $n \in \mathbb{N}$. By [9, Lemma 1.5.10], since $I \nsubseteq \cup_{\mathfrak{p} \in \operatorname{Ass}(N) \backslash V(l)} \mathfrak{p}$, there exists a homogeneous element $a_{1}$ in $I \backslash \cup_{\mathfrak{p} \in \operatorname{Ass}(N) \backslash V(I)} \mathfrak{p}$. Again, since

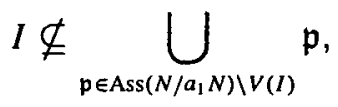

continuing in this way one can obtain the required homogeneous $I$-filter regular sequence $a_{1}, \ldots, a_{n}$ on $N$. 
REMARK 1. Note that if $a_{1}, \ldots, a_{k}$ is a homogeneous $I$-filter regular sequence on $N$ then, for all $i=1, \ldots, k$,

$$
\operatorname{Supp}_{R}\left(\frac{\left(a_{1}, \ldots, a_{i-1}\right) N:_{N} a_{i}}{\left(a_{1}, \ldots, a_{i-1}\right) N}\right) \subseteq V(I)
$$

Therefore, by [15, Proposition 1.2] (or [20, Lemma 3.4]), we have the following isomorphisms

$$
H_{l}^{i}(N) \cong \begin{cases}H_{\left(a_{1}, \ldots, a_{k}\right)}^{i}(N) & \text { for } 0 \leq i<k \\ H_{I}^{i-k}\left(H_{\left(a_{1}, \ldots, a_{k}\right)}^{k}(N)\right) & \text { for } k \leq i\end{cases}
$$

In the following proposition we show that the above isomorphisms are homogeneous. Let $R^{\prime}=\bigoplus_{n \in N_{0}} R_{n}^{\prime}$ be a second $\mathbb{N}_{0}$-graded commutative Noetherian ring. By [8, Definition 12.2.1], we say that the covariant functor $T: C(R) \longrightarrow C\left(R^{\prime}\right)$ has *restriction properties if

(i) whenever $M$ is a graded $R$-module, the $R^{\prime}$-module $T(M)$ is graded, and

(ii) the gradings in (i) are such that if $f: M \longrightarrow N$ is a homogeneous homomorphism of graded $R$-modules then $T(f): T(M) \longrightarrow T(N)$ is homogeneous.

PROPOSITION 2.2. Let I be a homogeneous ideal of a graded ring $R$. Suppose $k>1$ and $a_{1}, \ldots, a_{k}$ is a homogeneous $I$-filter regular sequence on $N$. Then there are homogeneous isomorphisms

$$
H_{I}^{i}(N) \cong \begin{cases}H_{\left(a_{1}, \ldots, a_{k}\right)}^{i}(N) & \text { for } 0 \leq i<k, \\ H_{I}^{i-k}\left(H_{\left(a_{1}, \ldots, a_{k}\right)}^{k}(N)\right) & \text { for } k \leq i .\end{cases}
$$

Proof. Let $i$ be an integer such that $0 \leq i<k$ and, for every graded $R$-module $N$, consider the natural grading on $H_{I}^{i}(N)$. Then we can define a grading on $H_{\left(a_{1}, \ldots, a_{k}\right)}^{i}(N)$ such that the isomorphism $H_{I}^{i}(N) \cong H_{\left(a_{1}, \ldots, a_{k}\right)}^{i}(N)$ is homogeneous. In the case $0 \leq i<k$; with respect to these gradings, the negative strongly connected sequence of functors $\left(H_{\left(a_{1}, \ldots, a_{k}\right)}^{i}\right)_{0 \leq i<k}$ has the ${ }^{*}$ restriction property. Similarly, in the case $k \leq i,\left(H_{\left(a_{1}, \ldots, a_{k}\right)}^{k}\right)_{k \leq i}$ and so $\left(H_{l}^{i-k}\left(H_{\left(a_{1}, \ldots, a_{k}\right)}^{k}\right)\right)_{k \leq i}$ have the *restriction property. Now, it follows from [8, Theorem 12.3.5] that these gradings coincide with the natural ones. Thus, the desired isomorphisms are homogeneous, as required.

REMARK 2: (See [6, Remark 3.2]) Assume that $\left(R_{0}, \mathrm{~m}_{0}\right)$ is a local ring. Set $R_{0}^{\prime}=R_{0}[x]_{\mathrm{m}_{0} R_{0}[x]}$, where $x$ is an indeterminate. Then $R_{0}^{\prime}$ is a faithfully flat Noetherian local $R_{0}$-algebra whose maximal ideal is $\mathrm{m}_{0}^{\prime}=\mathfrak{m}_{0} R_{0}^{\prime}$. Put $R^{\prime}=R_{0}^{\prime} \otimes_{R_{0}} R=$ $\bigoplus_{n \in N_{0}} R_{0}^{\prime} \otimes_{R_{0}} R_{n}$. Then $R^{\prime}$ is a positively standard graded ring, faithfully flat as an $R$-algebra. where $R_{+}^{\prime}=R_{+} R^{\prime}$. For every finitely generated graded $R$-module 
$L=\bigoplus_{n \in \mathbb{N}_{0}} L_{n}$, set $L^{\prime}=R^{\prime} \otimes_{R} L=R_{0}^{\prime} \otimes_{R_{0}} L=\bigoplus_{n \in \mathbb{N}_{0}} R_{0}^{\prime} \otimes_{R_{0}} L_{n}$, which is a finitely generated $R^{\prime}$-module. Since $R_{0}^{\prime}$ is an $R_{0}$-flat, in view of the statement (iii) in Section 1, the faithful $R_{0}$-flatness of $R_{0}^{\prime}$ gives rise to an isomorphism of $R_{0}^{\prime}$-modules $H_{R_{+}^{\prime}}^{i}\left(M^{\prime}, N^{\prime}\right)_{n} \cong H_{R_{+}}^{i}(M, N)_{n} \otimes_{R_{+}} R_{0}^{\prime}$ for all $i \in \mathbb{N}_{0}$ and $n \in \mathbb{Z}$. This, together with [19, Theorem (23.2)(ii)], shows that

$$
\operatorname{Ass}_{R_{0}}\left(H_{R_{+}}^{i}(M, N)_{n}\right)=\left\{\mathfrak{p}_{0}^{\prime} \cap R_{0} \mid \mathfrak{p}_{0}^{\prime} \in \operatorname{Ass}_{R_{0}^{\prime}}\left(H_{R_{+}^{\prime}}^{i}\left(M^{\prime}, N^{\prime}\right)_{n}\right)\right\} .
$$

LEMMA 2.3. Suppose $R=\bigoplus_{n \in \mathbb{N}_{0}} R_{n}$ is a positively standard graded Noetherian ring and that the base ring $R_{0}$ is local. Let Mand $N$ be finitely generated and graded $R$-modules and let $k$ be a positive integer such that $H_{R_{+}}^{i}(N)$ is finitely generated for every $i<k$. Then $\operatorname{Ass}_{R_{0}}\left(H_{R_{+}}^{k}(M, N)_{n}\right)$ is asymptotically stable as $n \longrightarrow-\infty$.

PROOF. According to Remark 2, we may replace $R, M$ and $N$ respectively by $R^{\prime}, M^{\prime}$ and $N^{\prime}$ and hence assume that the residue field $R_{0} / \mathrm{m}_{0}$ is infinite. By Proposition 2.1, there exists a homogeneous $R_{+}$-filter regular sequence $a_{1}, \ldots, a_{k+1}$ on $N$.

Now, set $S_{0}=N$ and $S_{i}=H_{\left(a_{1}, \ldots, a_{i}\right)}^{i}(N)$ for $i=1, \ldots, k+1$. In view of [8, Exercise 1.1.2] and Proposition 2.2, we have the homogeneous isomorphisms

$$
H_{\left(a_{i}\right)}^{0}\left(S_{i-1}\right) \cong H_{\left(a_{1}, \ldots, a_{i}\right)}^{0}\left(S_{i-1}\right) \cong H_{R_{+}}^{i-1}(N) \text { and } \quad H_{\left(a_{i}\right)}^{1}\left(S_{i-1}\right) \cong H_{\left(a_{1}, \ldots, a_{i}\right)}^{i}(N) .
$$

So, by [8, Exercise 12.4.2], we obtain the following exact sequence of homogeneous homomorphisms

$$
0 \longrightarrow H_{R_{+}}^{i-1}(N) \longrightarrow S_{i-1} \stackrel{f_{i}}{\longrightarrow}\left(S_{i-1}\right)_{a_{i}} \longrightarrow S_{i} \longrightarrow 0
$$

for all $i=1, \ldots, k+1$, where $f_{i}$ is considered as canonical map. We may now obtain two homogeneous short exact sequences as follows

$$
\begin{aligned}
& 0 \longrightarrow \operatorname{Im} f_{i} \longrightarrow\left(S_{i-1}\right)_{a_{i}} \longrightarrow S_{i} \longrightarrow 0 \text { and } \\
& 0 \longrightarrow H_{R_{+}}^{i-1}(N) \longrightarrow S_{i-1} \longrightarrow \operatorname{Im} f_{i} \longrightarrow 0 .
\end{aligned}
$$

On the other hand, since the multiplication by $a_{i}$ provides an automorphism on $\left(S_{i-1}\right)_{a_{i}}$ and $H_{R_{+}}^{t}\left(M,\left(S_{i-1}\right)_{a_{i}}\right)$ is $R_{+}$-torsion, we have $H_{R_{+}}^{t}\left(M,\left(S_{i-1}\right)_{a_{i}}\right)=0$ for all $t \in \mathbb{N}_{0}$ and $i=1, \ldots, k+1$. Hence, by applying the functor $H_{R_{+}}^{t}(M,-)$ on $(2.1)$, we obtain the homogeneous isomorphism

$$
H_{R_{+}}^{t}\left(M, S_{i}\right) \cong H_{R_{+}}^{i+1}\left(M, \operatorname{Im} f_{i}\right) \quad \text { for all } t \in \mathbb{N}_{0} .
$$

Also, by applying the functor $H_{R_{+}}^{t}(M,-)$ on (2.2) together with (2.3), we obtain the homogeneous exact sequence

$$
H_{R_{+}}^{t}\left(M, H_{R_{+}}^{i-1}(N)\right) \rightarrow H_{R_{+}}^{t}\left(M, S_{i-1}\right) \rightarrow H_{R_{+}}^{t-1}\left(M, S_{i}\right) \rightarrow H_{R_{+}}^{t+1}\left(M, H_{R_{+}}^{i-1}(N)\right)
$$


for all $t \in \mathbb{N}$ and $i=1, \ldots, k+1$. Now, in view of [14, Lemma 3.1], the graded $R$-module $H_{R_{+}}^{t}\left(M, H_{R_{+}}^{i-1}(N)\right)$ has only finitely many nonzero components, so there exists $n_{1} \in \mathbb{Z}$ such that for all $n \leq n_{1}$,

$$
H_{R_{+}}^{\prime}\left(M, H_{R_{+}}^{i-1}(N)\right)_{n}=0=H_{R_{+}}^{t+1}\left(M, H_{R_{+}}^{i-1}(N)\right)_{n} .
$$

This implies that there is a homogeneous isomorphism

$$
H_{R_{+}}^{t}\left(M, S_{i-1}\right)_{n} \cong H_{R_{+}}^{i-1}\left(M, S_{i}\right)_{n}
$$

for all $t \in \mathbb{N}, n \leq n_{1}$ and $i=1, \ldots, k+1$. Hence we obtain the following homogeneous isomorphism

$$
H_{R_{+}}^{k}(M, N)_{n} \cong H_{R_{+}}^{0}\left(M, S_{k}\right)_{n}
$$

for all $n \leq n_{1}$ and so it is enough for us to show that $\operatorname{Ass}_{R_{0}}\left(H_{R_{+}}^{0}\left(M, S_{k}\right)_{n}\right)$ is asymptotically stable as $n \longrightarrow-\infty$. In order to do this, we claim that there is a homogeneous isomorphism $H_{R_{+}}^{0}\left(M, S_{k}\right) \cong H_{R_{+}}^{0}\left(M, H_{R_{+}}^{k}(N)\right)$. To show this, consider the homogeneous exact sequence

$$
0 \longrightarrow H_{R_{+}}^{k}(N) \longrightarrow S_{k} \longrightarrow\left(S_{k}\right)_{a_{k+1}}
$$

to deduce the homogeneous exact sequence

$$
0 \longrightarrow H_{R_{+}}^{0}\left(M, H_{R_{+}}^{k}(N)\right) \longrightarrow H_{R_{+}}^{0}\left(M, S_{k}\right) \longrightarrow H_{R_{+}}^{0}\left(M,\left(S_{k}\right)_{a_{k+1}}\right)=0 .
$$

Thus, by [6, Lemma 5.4] in view of [9, Exercise 1.2.28], we conclude that

$$
\begin{aligned}
\operatorname{Ass}_{R_{0}}\left(H_{R_{+}}^{k}(M, N)_{n}\right) & =\operatorname{Ass}_{R_{0}}\left(H_{R_{+}}^{0}\left(M, S_{k}\right)_{n}\right)=\operatorname{Ass}_{R_{0}}\left(H_{R_{+}}^{0}\left(M, H_{R_{+}}^{k}(N)\right)_{n}\right) \\
& =\operatorname{Ass}_{R_{0}}\left(\operatorname{Hom}_{R}\left(M, H_{R_{+}}^{k}(N)\right)_{n}\right),
\end{aligned}
$$

where the last equality holds by statement (iv) of Section 1 . Now the last term is asymptotically stable as $n \longrightarrow-\infty$ and the proof is complete.

In order to state the main theorem, we need the concept of the finiteness dimension of an $R$-module. Let $N$ be a finitely generated and graded $R$-module. Recall that the finiteness dimension of $N$ relative to $R_{+}$is defined by

$$
f_{R_{+}}(N)=\inf \left\{i \in \mathbb{N} \mid H_{R_{+}}^{i}(N) \text { is not finitely generated }\right\} .
$$

As, by [8, Proposition 15.1.5], $H_{R_{+}}^{i}(N)_{n}$ is finitely generated for all $i \in \mathbb{N}_{0}$ and all $n \in \mathbb{Z}$ and vanishes for all $n \gg 0$, we may write

$$
\begin{aligned}
f_{R_{+}}(N) & =\inf \left\{i \in \mathbb{N}_{0} \mid \#\left\{n \leq 0 \mid H_{R_{+}}^{i}(N)_{n} \neq 0\right\}=\infty\right\} \\
& =\inf \left\{i \in \mathbb{N}_{0} \mid \#\left\{n \in \mathbb{Z} \mid H_{R_{+}}^{i}(N)_{n} \neq 0\right\}=\infty\right\} .
\end{aligned}
$$


THEOREM 2.4. Let $M$ and $N$ be finitely generated and graded $R$-modules and let $f=f_{R_{+}}(N) \in \mathbb{N}$. Then Ass $_{R_{0}}\left(H_{R_{+}}^{f}(M, N)_{n}\right)$ is asymptotically stable as $n \longrightarrow-\infty$. Moreover, $H_{R_{+}}^{f}(M, N)$ is an asymptotically gap free $R$-module.

Proof. It is well known that, for all $i \in \mathbb{N}_{0}$,

$$
\mathfrak{p} \in \operatorname{Ass}_{R}\left(H_{R_{+}}^{i}(M, N)\right) \quad \text { if and only if } \mathfrak{p} \cap R_{0} \in \operatorname{Ass}_{R_{0}}\left(H_{R_{+}}^{i}(M, N)\right),
$$

and that

$$
\operatorname{Ass}_{R_{0}}\left(H_{R_{+}}^{i}(M, N)\right)=\bigcup_{n \in \mathbb{Z}} \operatorname{Ass}_{R_{0}}\left(H_{R_{+}}^{i}(M, N)_{n}\right) .
$$

Also, in view of [16, Theorem 2.3], $\operatorname{Ass}_{R}\left(H_{R_{+}}^{f}(M, N)\right)$ is finite. So,

$$
\mathscr{A}=\bigcup_{n \in \mathbb{Z}} \operatorname{Ass}_{R_{0}}\left(H_{R_{+}}^{f}(M, N)_{n}\right)
$$

is finite. Suppose that $\mathfrak{p}_{0} \in \mathscr{A}$. Then $H_{\left(R_{\left.\mathfrak{p}_{0}\right)_{+}}\right)}^{i}\left(N_{\mathfrak{p}_{0}}\right)$ is a finitely generated $R_{\mathfrak{p}_{0}}$-module for all $i<f$. Now, Lemma 2.3 implies that

$$
\operatorname{Ass}_{\left(R_{0}\right)_{\mathfrak{p}_{0}}}\left(H_{\left(R_{\mathfrak{p}_{0}}\right)_{+}}^{f}\left(M_{\mathfrak{p}_{0}}, N_{\mathrm{p}_{0}}\right)_{n}\right)
$$

is asymptotically stable as $n \longrightarrow-\infty$. As $\mathfrak{p}_{0} \in \operatorname{Ass}_{R_{0}}\left(H_{R_{+}}^{f}(M, N)_{n}\right)$ if and only if

$$
\mathfrak{p}_{0}\left(R_{0}\right)_{\mathfrak{p}_{0}} \in \operatorname{Ass}_{\left(R_{0}\right)_{\mathfrak{p}_{0}}}\left(H_{\left(R_{\mathfrak{p}_{0}}\right)_{+}}^{f}\left(M_{\mathfrak{p}_{0}}, N_{\mathfrak{p}_{0}}\right)_{n}\right) \text {, }
$$

it follows that $\operatorname{Ass}_{R_{0}}\left(H_{R_{+}}^{f}(M, N)_{n}\right)$ is asymptotically stable as $n \longrightarrow-\infty$, as required in the first statement. The second statement is now obvious.

Corollary 2.5. (See [6, Proposition 5.6].) Suppose that $N$ is a finitely generated and graded $R$-module and let $f=f_{R_{+}}(N) \in \mathbb{N}$. Then $\operatorname{Ass}_{R_{0}}\left(H_{R_{+}}^{f}(N)_{n}\right)$ is asymptotically stable as $n \longrightarrow-\infty$ and $H_{R_{+}}^{f}(N)$ is an asymptotically gap free $R$-module.

\section{Acknowledgements}

The first author was partially supported by a grant from the Institute for Studies in Theoretical Physics and Mathematics (IPM) Iran (No. 84130025)

\section{References}

[1] J. Asadollahi, K. Khashyarmanesh and Sh. Salarian, 'On the finiteness properties of the generalized local cohomology modules', Comm. Algebra 30 (2002), 859-867. 
[2] M. H. Bijan-Zadeh, 'A comımon generalization of local cohomology theories', Glasg. Math. J. 21 (1980), 173-181.

[3] M. Brodmann, 'Asymptotic behaviour of cohomology: tameness, supports and associated primes', in: Commutative algebra and algebraic geometry, Contemp. Math. 390 (Amer. Math. Soc., Providence, RI, 2005) pp. 31-61.

[4] M. Brodmann, S. Fumasoli and C. S. Lim, 'Low-codimensional associated primes of graded components of local cohomology modules', J. Algebra 275 (2004), 867-882.

[5] M. Brodmann, S. Fumasoli and R. Tajarod, 'Local cohomology over homogeneous rings with one-dimensional local bast ring', Proc. Amer. Math. Soc. 131 (2003), 2977-2985.

[6] M. Brodmann and. M. Hillus, 'Cohomological patterns of coherent sheaves over projective schemes', J. Pure Appl. Al' ebra 172 (2002), 165-182.

[7] M. Brodmann, M. Katzman and R. Y. Sharp, 'Associated primes of graded components of local cohomology modules', Trai:s. Amer. Math. Soc. 354 (2002), 4261-4283.

[8] M. Brodmann and R. Y. Sharp, Local cohomology - an algebraic introduction with geometric applications, volume 60 of Cambridge studies in advanced mathematics (Cambridge University Press, 1998).

[9] W. Bruns and J. Herzog, Cohen-Macaulay rings, volume 39 of Cambridge studies in advanced mathematics (Cambridge University Press, 1993).

[10] J. Herzog, 'Komplexe, auflösungen und dualität in der lokalen algebra', Preprint ???? , 1974.

[11] J. Herzog and N. Zamani, 'Duality and vanishing of generalized local cohomology', Arch. Math. (Basel) 81 (2003), 512-519.

[12] S. Huckaba and T. Marley, 'On associated graded rings of normal ideals', J. Algebra 222 (1999), 146-163.

[13] M. Katzman and R. Y. Sharp, 'Some properties of top graded local cohomology modules', $J$. Algebra 259 (2003), 599-612.

[14] K. Khashyarmanesh, 'Associated primes of graded components of gneralized local cohomology modules', Comm. Algebra 33 (2005), 3081-3090.

[15] K. Khashyarmanesh and Sh. Salarian, 'Filter regular sequences and the finiteness of local cohomology modules', Comm. Algebra 26 (1998), 2483-2490.

[16] K. Khashyarmanesh, M. Yassi and A. Abbasi, 'Filter regular sequences and generalized local cohomology modules', Comm. Algebra 32 (2004), 253-259.

[17] C. S. Lim, 'Graded local cohomology modules and their associated primes: the Cohen-Macaulay case', J. Pure Appl. Algebra 185 (2003), 225-238.

[18] _ _ ' 'Graded local cohomology modules and their associated primes', Comm. Algebra 32 (2004), 727-745.

[19] H. Matsumura, Commutative Ring Therory, volume 8 of Cambridge studies in advanced mathematics (Cambridge University Press, 1986).

[20] U. Nagel and P. Schenzel, 'Cohomological annihilators and Castelnuovo-Mumford regularity', in: Commutative algebra: syzygies, multiplicities, and birational algebra (South Hadley, MA, 1992), Contemp. Math. 159 (Amer. Math. Soc., Providence, RI, 1994) pp. 307-328.

[21] N. Suzuki, 'On the generalized local cohomology and its duality', J. Math. Kyoto Univ. (JAKYAZ) 18-1 (1978), 71-85.

[22] S. Yassemi, 'Generalized section functor', J. Pure Appl. Algebra 95 (1994), 103-119.

[23] S. Yassemi, L. Khatami and T. Sharif, 'Associated primes of generalized local cohomology modules', Comm. Algebra 30 (2002), 327-330. 
Fedowsi University of Mashhad Department of Mathematics

P.O. Box 1159-91775

Mashhad

Iran

and

Institute for Studies in

Theoretical Physics and Mathematics

P.O. Box 19395-5746

Tehran

Iran

e-mail: khashyar@ipm.ir
Guilan University Department of Mathematics P.O. Box 41335-1914 Rasht

Iran. e-mail: aabbasi@guilan.ac.ir 
